$M P E$ - Volume 2, pp. 57-61

Reprints available directly from the publisher

Photocopying permitted by license only
(C) 1996 OPA (Overseas Publishers Association) Amsterdam B.V. Published in The Netherlands

Under license by Gordon and Breach Science

Publishers SA

Printed in Malaysia

\title{
A NOTE ON THE CONVERGENCE OF QUANTIZERS
}

\author{
ERIC B. HALL \\ Department of Electrical Engineering, Southern Methodist University, P.O. Box 750338, \\ Dallas, Texas 75275-0338
}

Accepted for publication by Dr. V. Lakshmikantham

(Received March 13, 1995)

Several questions concerning the convergence of quantized estimators are considered based upon points of accuracy. Examples are considered in which quantizers converge and in which they fail to converge, and points of accuracy are shown to provide explanations for both types of behavior.

KEYWORDS: Quantization; convergence; approximation; points of accuracy AMS 94A99

\section{INTRODUCTION}

Many classical techniques in engineering involve the observation of continuous-time random processes. Unfortunately, one is seldom (if ever) able to observe a process continuously. Thus, in practice, observations are generally quantized. In this way, quantization becomes a bridge between engineering theory and engineering practice. In this paper we will consider several questions regarding the convergence of quantizers, and we will provide answers, to these questions based upon a useful tool of analysis that we will call a point of accuracy.

\section{DEFINITIONS}

Consider a finite subset $M=\left\{m_{1}, \ldots, m_{N}\right\}$ of $\mathbb{R}$ and a function $f$ mapping $\mathbb{R}$ to $M$. The function $f$ is called an $N$-level quantizer if $f\left(m_{i}\right)=m_{i}$ for each positive integer $i \leq N$. (A Borel measurable quantizer is sometimes called a round-off map.) For a quantizer $f$, the collection of sets $f^{-1}\left(m_{i}\right)$ for $1 \leq i \leq N$ is called the partition of $\mathbb{R}$ induced by the quantizer $f$. Note that a quantizer (on $\mathbb{R}$ ) is simply a mapping of $\mathbb{R}$ to a finite subset $M$ of $\mathbb{R}$ such that the map restricted to $M$ is the identity function. (Although this definition is very commonly used, it is typically much too general to be useful in any theoretical sense. See, for example, the work in [1].)

Consider a sequence $\left\{f_{n}\right\}_{n=1}^{\infty}$ of quantizers, and recall that the diameter of a subset $A$ of $\mathbb{R}$ is denoted by diam $A$ and is defined via diam $A=\sup _{x, y \in A}|x-y|$. A point $x \in \mathbb{R}$ will be called $a$ point of accuracy with respect to the sequence $\left\{f_{n}\right\}_{n=1}^{\infty}$ of quantizers if $\lim _{n \rightarrow \infty}$ 
$\operatorname{diam} f_{n}^{-1}\left(f_{n}(x)\right)=0$. It will be shown below that points of accuracy may be used to explain why some sequences of quantizers exhibit many desired convergence properties and why others are extremely pathological.

As usual, for a function $f$ mapping a nonempty set $\Omega$ to $\mathbb{R}$, we will let $\sigma(f)$ denote $f^{-1}(\boldsymbol{B}(\mathbb{R}))$. A sequence $\left\{f_{n}\right\}_{n=1}^{\infty}$ of quantizers is said to be a (strictly) increasing sequence if $\sigma\left(f_{n}\right)$ is a (proper) subset of $\sigma\left(f_{n+1}\right)$ for each positive integer $n$.

\section{DEVELOPMENT}

Although many results in quantization are intuitively appealing, such is not always the case. Indeed, as the following result from [1] indicates, intuition can fail dramatically in certain instances.

Theorem 1 For any positive integer $k$ and any integer $N>1$, a probability space, a Gaussian random vector $X$ defined on the space taking values in $\mathbb{R}^{k}$ and having a positive definite covariance matrix, and an $N$-level quantizer $Q$ exist such that the random vector $Q(X)$ takes on each of the $N$ values in its range with equal probability and such that $X$ and $Q(X)$ are independent.

Notice that neither an arbitrarily high number of equally probable levels nor the ubiquitous Gaussian assumption is enough to ensure even the modest desire that the output of a quantizer will be statistically dependent upon the input! The following corollary to Theorem 1 shows that a quantization model that ignores points of accuracy may be quite pathological.

Corollary 1 There exists a positive variance Gaussian random variable $X$ and an increasing sequence $\left\{f_{n}\right\}_{n=1}^{\infty}$ of quantizers such that the sequence has no points of accuracy and such that $X$ and $f_{n}(X)$ are independent for each positive integer $n$.

Proof. Let $m$ be a positive integer. As provided by Example 6.9 of [2], let $\left\{S_{i}: i \leq m\right\}$ be a partition of $\mathbb{R}$ into saturated non-Lebesgue measurable subsets. (An example of such a subset $B$ of $\mathbb{R}$ is a Bernstein set, which has the unusual property that $B$ and $B^{c}$ each have a nonempty intersection with every uncountable closed subset of $\mathbb{R}$.) Note that, for $1<$ $n \leq m$,

$$
T_{n}=\bigcup_{j=n}^{m} S_{j}
$$

is also a saturated non-Lebesgue measurable set. Let $\alpha_{i} \in S_{i}$ for each integer $0<i \leq m$, and let

$$
f_{n}(x)=\left\{\begin{array}{c}
\alpha_{n} I_{T_{n}}(x)+\sum_{i=1}^{n-1} \alpha_{i} I_{S_{i}}(x) \text { if } 1 \leq n \leq m \\
\sum_{i=1}^{m} \alpha_{i} I_{S_{i}}(x) \quad \text { if } n>m,
\end{array}\right.
$$


where $\mathrm{I}_{A}$ is the indicator function of the set $A$. Note that the $f_{n}$ 's form an increasing sequence of quantizers. Further, no point of $\mathbb{R}$ is a point of accuracy since the $S_{i}$ 's are unbounded. The remaining part of the proof follows as in [1].

In a more positive vein, the following results about sequences of strictly increasing Borel measurable quantizers for which every real number is a point of accuracy are presented in [3].

Lemma 1 Consider a sequence $\left\{f_{n}\right\}_{n=1}^{\infty}$ of strictly increasing Borel measurable quantizers for which every point in $\mathbb{R}$ is a point of accuracy. The smallest $\sigma$-algebra containing every set in $\sigma\left(f_{n}\right)$ for every positive integer $n\left(\right.$ denoted by $\bigvee_{n=1}^{\infty} \sigma\left(f_{n}\right)$ ) is $\boldsymbol{B}(\mathbb{R})$.

Lemma 2 Consider a sequence $\left\{f_{n}\right\}_{n=1}^{\infty}$ of strictly increasing Borel measurable quantizers for which every point in $\mathbb{R}$ is a point of accuracy. Further, consider a random variable $X$ defined on a probability space $(\Omega, \mathscr{F}, P)$. It then follows that

$$
\bigvee_{n=1}^{\infty} \sigma\left(f_{n}(X)\right)=\sigma(X)
$$

Theorem 2 Consider a probability space $\left(\Omega, \mathscr{F}_{F}, P\right)$, a strictly increasing sequence $\left\{f_{n}\right\}_{n=1}^{\infty}$ of Borel measurable quantizers for which every point in $\mathbb{R}$ is a point of accuracy, a random variable $Y$ defined on $\left(\Omega, F_{F}, P\right)$, and a random variable $X \in L_{p}\left(\Omega, F_{F}, P\right)$ for some $p \in[1, \infty)$. It then follows that $E\left[X \mid f_{n}(Y)\right]$ converges to $E[X \mid Y]$ almost surely and in $L_{p}$.

Consider a sequence $\left\{f_{n}\right\}_{n=1}^{\infty}$ of quantizers, and let $A$ denote the set of all real numbers $x$ such that $x$ is a point of accuracy for the sequence $\left\{f_{n}\right\}_{n=1}^{\infty}$. We have seen that if $A$ is empty then serious pathologies may occur and if $A$ is the real line then standard estimation techniques based upon conditioning may converge almost surely and in $L_{p}$ to the nonquantized estimate. That is, the work in [1] and in [3] has considered two different sides of the same coin. At this point, one might wonder if the conclusion of Lemma 1 holds when $A$ is a proper subset of $\mathbb{R}$. The following lemma provides an answer to this question for an important special case in which the sets in the induced partition are connected.

Lemma 3 Consider a sequence $\left\{f_{n}\right\}_{n=1}^{\infty}$ of strictly increasing quantizers such that

$$
\bigvee_{n=1}^{\infty} \sigma\left(f_{n}\right)=\boldsymbol{B}(\mathbb{R})
$$

and such that $f_{n}^{-1}\left(f_{n}(x)\right)$ is connected for each $x \in \mathbb{R}$ and each $n \in \mathbb{N}$. Every point in $\mathbb{R}$ is a point of accuracy for this sequence of quantizers.

Proof. Assume that there exists some point $z \in \mathbb{R}$ such that $z$ is not a point of accuracy for the $f_{n}$ 's. Then, since $f_{n}^{-1}\left(f_{n}(z)\right)$ is connected for each $n \in \mathbb{N}$, there exist distinct points $x$ and $y$ in $\mathbb{R}$ such that $f_{n}(x)=f_{n}(y)$ for all $n \in \mathbb{N}$. Let $\mathscr{B}$ be the subset of $\boldsymbol{B}(\mathbb{R})$ that consists of all sets of the form $A \cup\{x, y\}$ and $A \backslash\{x, y\}$ where $A \in B(\mathbb{R})$. Note that $\mathscr{B}$ is a $\sigma$-algebra 
and that $\mathscr{B}$ is a proper subset of $\boldsymbol{B}(\mathbb{R})$. Since $\bigvee_{n=1}^{\infty} \sigma\left(f_{n}\right)$ is a subset of $\mathscr{B}$ and is equal to $\boldsymbol{B}(\mathbb{R})$, it follows by contradiction that no such point $z$ can exist. Thus, every point in $\mathbb{R}$ is a point of accuracy, and the proof is completed.

The proof of Lemma 3 suggests the next result, which, under the additional requirement that the sets in the induced partition containing any particular point are eventually bounded, presents a condition that is both necessary and sufficient to ensure that each point in $\mathbb{R}$ is a point of accuracy.

Lemma 4 Consider a sequence $\left\{f_{n}\right\}_{n=1}^{\infty}$ of strictly increasing quantizers such that, for each $x \in \mathbb{R}, f_{n}^{-1}\left(f_{n}(x)\right)$ is connected for each $n \in \mathbb{N}$ and is bounded for some $n \in \mathbb{N}$. Let $A$ be the set of all points of accuracy for this sequence of quantizers. Then $A=\mathbb{R}$ if and only if given any distinct points $x$ and $y$ from $\mathbb{R}$ there exists some positive integer $m$ such that $f_{m}(x) \neq f_{m}(y)$.

Proof. Assume that $A=\mathbb{R}$. Fix $x \in \mathbb{R}$, let $A_{n}=f_{n}^{-1}\left(f_{n}(x)\right)$ for each $n \in \mathbb{N}$, and let $B=\cap_{n=1}^{\infty} A_{n}$. Note that $B$ is not empty since $x \in A_{n}$ for each $n \in \mathbb{N}$. If $B$ contains more than one point then diam $B>0$. Note, however, that diam $B \leq \operatorname{diam} A_{n}$ for each $n \in \mathbb{N}$ since $B \subset A_{n}$ for each $n \in \mathbb{N}$. This contradicts our assumption that diam $A_{n} \rightarrow 0$. Thus, $B$ contains only one point, which implies that there exists no point $y \neq x$ such that $f_{n}(x)$ $=f_{n}(y)$ for all $n \in \mathbb{N}$.

Now, assume that there exists no point $y \neq x$ such that $f_{n}(x)=f_{n}(y)$ for all $n \in \mathbb{N}$. Fix $z \in \mathbb{R}$, let $A_{n}=f_{n}^{-1}\left(f_{n}(z)\right)$ for each $n \in \mathbb{N}$, and let $B=\cap_{n=1}^{\infty} A_{n}$. Note that $z \in B$. If $x \neq z$ and $x \in B$ then $f_{n}(x)=f_{n}(z)$ for all $n \in \mathbb{N}$, which contradicts our assumption. Thus, $B=\{z\}$. Note that lim diam $A_{n}$ exists since the sequence is monotonic and, for large $n$, is bounded. Assume that this limit is positive. Then, since the $A_{n}$ 's are connected, there exists some point $x \neq z$ such that $x \in A_{n}$ for each $n \in \mathbb{N}$. This contradiction implies that $\lim _{n \rightarrow \infty} \operatorname{diam} A_{n}=0$, which, in turn, implies that $A=\mathbb{R}$, and the proof is completed.

Lemma 4 is intuitively appealing since it relates points of accuracy to a condition that requires the sequence of quantizers to eventually distinguish any two distinct points. Further, Lemmas 3 and 4 are each useful for the standard "level-set" quantizers. Note, however, that they need not hold in the general case since, in general, $A_{n} \rightarrow\{x\}$ need not imply that diam $A_{n} \rightarrow 0$ even when the sets of interest are bounded. For example, if $A_{n}$ $=\{0\} \cup(1,1+1 / n)$ then $A_{n} \rightarrow\{0\}$ yet diam $A_{n} \rightarrow 1$.

Finally, combining Lemma 4 with Theorem 2, we obtain the following result that provides both a simple test and a simple explanation of the convergence properties of quantizers in a very common setting. It is instructive to contrast this result with that of Corollary 1 , in which there were no points of accuracy.

Theorem 3 Consider a strictly increasing sequence $\left\{f_{n}\right\}_{n=1}^{\infty}$ of Borel measurable quantizers such that, for each $x \in \mathbb{R}, f_{n}^{-1}\left(f_{n}(x)\right)$ is connected for each $n \in \mathbb{N}$ and is bounded for some $n \in \mathbb{N}$. Assume that if $x \neq y$ then $f_{m}(x) \neq f_{m}(y)$ for some $m \in \mathbb{N}$. Consider, also, a probability space $\left(\Omega, \mathscr{F}_{F}, P\right)$, a random variable $Y$ defined on $\left(\Omega, \mathscr{F}_{F}, P\right)$, and a random variable $X \in L_{p}\left(\Omega, \mathscr{F}_{F}, P\right)$ for some $p \in[1, \infty)$. It then follows that $E\left[X \mid f_{n}(Y)\right]$ converges to $E[X \mid Y]$ almost surely and in $L_{p}$. 


\section{CONCLUSION}

A method for modeling quantization effects based upon points of accuracy was introduced. It was shown that serious problems may occur when points of accuracy do not exist. Further, it was shown that when every point is a point of accuracy, many techniques in estimation and decision theory involving conditional expectation may be attained asymptotically via techniques based upon quantized observations. In addition, for a very common setting, a condition was presented that is both necessary and sufficient to ensure that each point in $\mathbb{R}$ is a point of accuracy.

\section{References}

1. E. B. Hall and G. L. Wise, A Result on Multidimensional Quantization, Proceedings of the American Mathematical Society, 118 (1993), 609-613.

2. G. L. Wise and E. B. Hall, Counterexamples in Probability and Real Analysis, Oxford University Press, New York, 1993.

3. J. M. Morrison and G. L. Wise, An Asymptotic Property of Nonlinear Estimators Arising as Solutions to a Certain Class of Convex Programming Problems, Systems and Control Letters, 7 (1986), 225-232. 


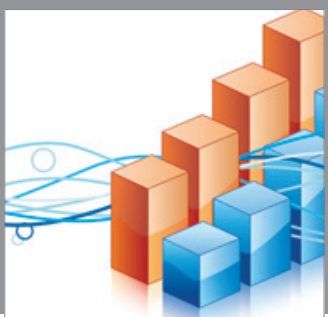

Advances in

Operations Research

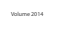

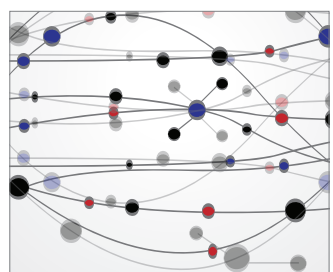

\section{The Scientific} World Journal
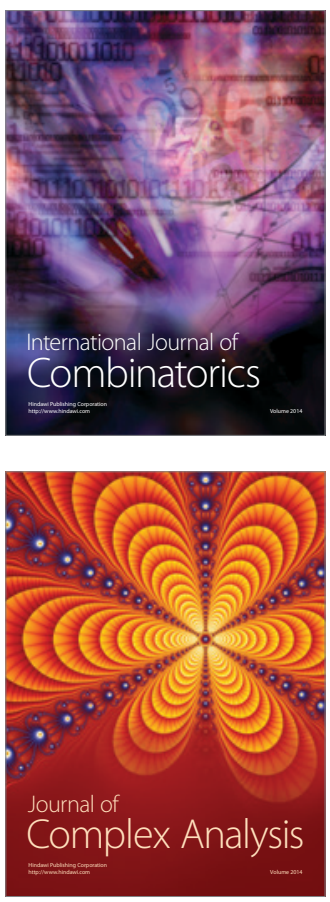

International Journal of

Mathematics and

Mathematical

Sciences
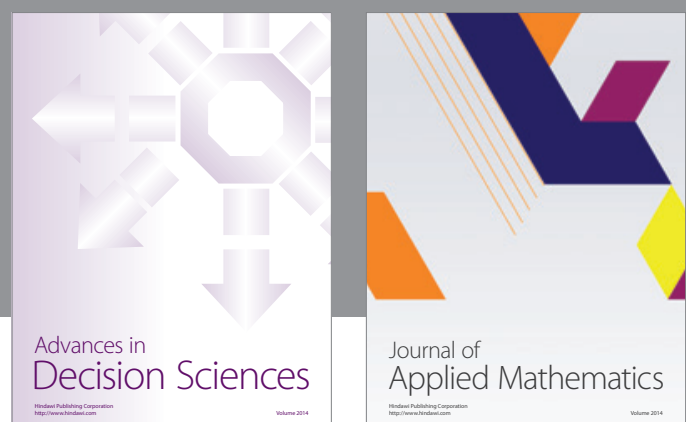

Journal of

Applied Mathematics
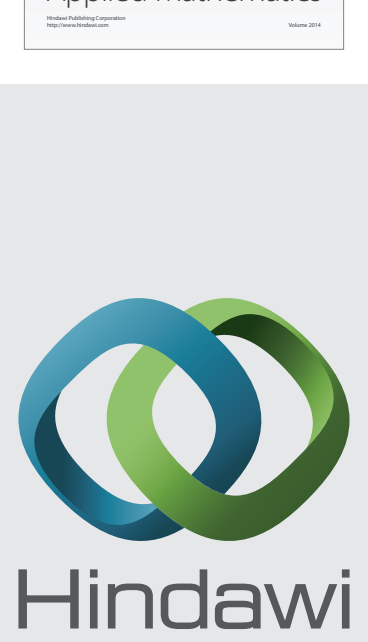

Submit your manuscripts at http://www.hindawi.com
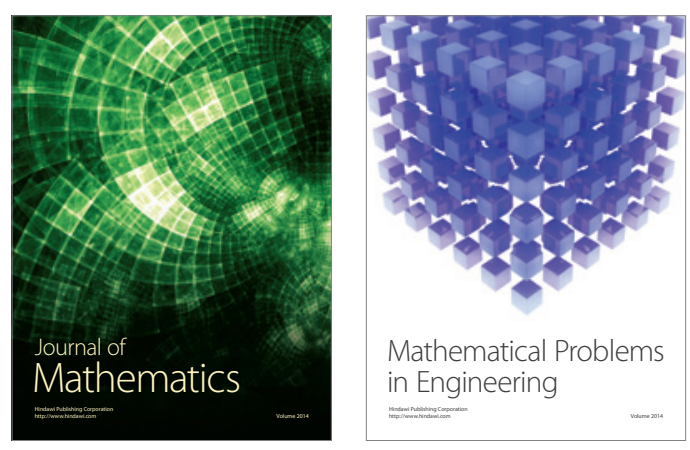

Mathematical Problems in Engineering
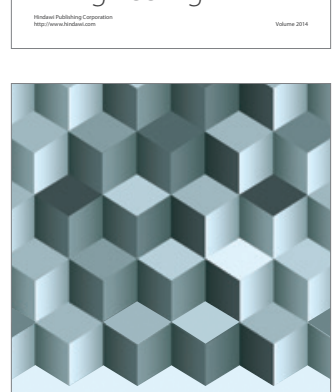

Journal of

Function Spaces
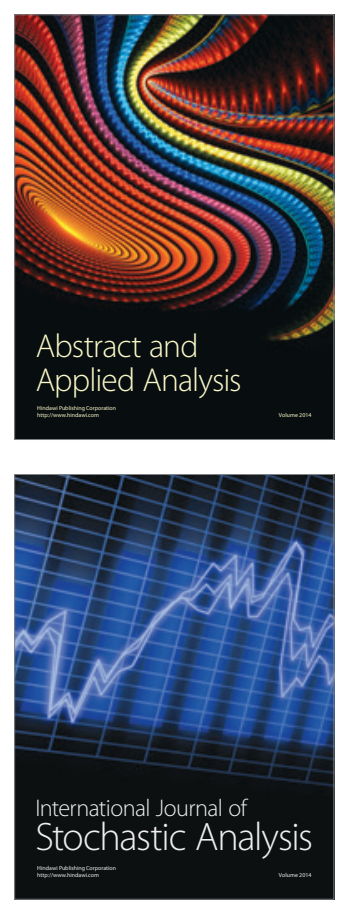

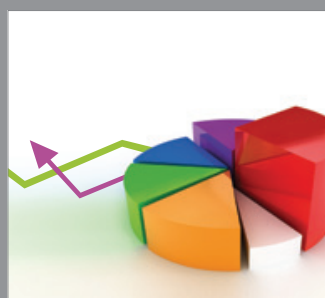

ournal of

Probability and Statistics

Promensencen
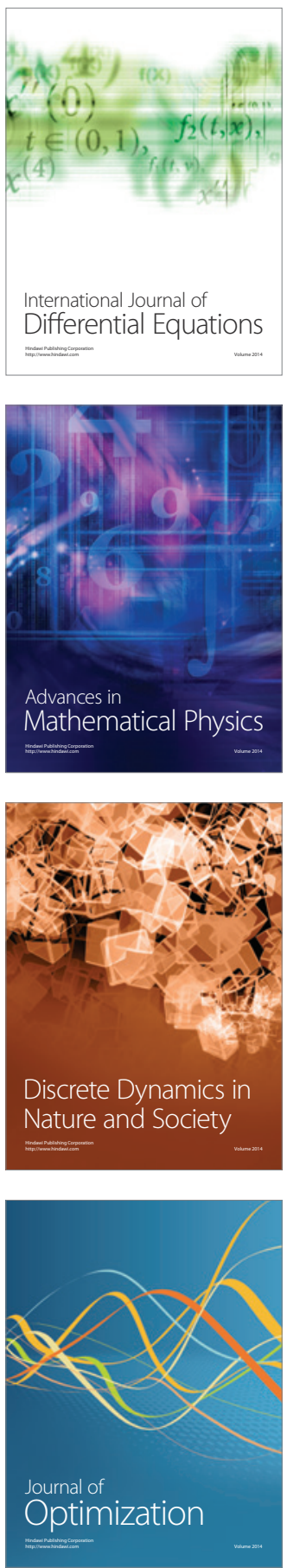